\title{
Global Analysis of Solutions of a New Class of Rational Difference Equation
}

\author{
0. Moaaz ${ }^{1}$, E. M. Elabbasy ${ }^{1}$, Sh. Alsaeed ${ }^{2}$ \\ ${ }^{1}$ Department of Mathematics, Faculty of Science, Mansoura University, Mansoura, Egypt \\ ${ }^{2}$ Department of Mathematics, The Faculty of Education, Al al-Bayt University, Mafraq, Jordan \\ Email:emelabbasy@mans.edu.eg,o_moaaz@mans.edu.eg,shaimaa.alsaeed@yahoo.com
}

How to cite this paper: Moaaz, O., Elabbasy, E.M. and Alsaeed, S. (2017) Global Analysis of Solutions of a New Class of Rational Difference Equation. American Journal of Computational Mathematics, 7, 495-503.

https://doi.org/10.4236/ajcm.2017.74036

Received: July 28, 2017

Accepted: December 16, 2017

Published: December 19, 2017

Copyright () 2017 by authors and Scientific Research Publishing Inc. This work is licensed under the Creative Commons Attribution International License (CC BY 4.0). http://creativecommons.org/licenses/by/4.0/

\begin{abstract}
The study suggests asymptotic behavior of the solution to a new class of difference equations: $\psi_{\eta+1}=a+\sum_{i=0}^{k} b_{i} \frac{\psi_{\eta-(2 i+1)}}{\alpha \psi_{\eta-2 i}+\beta \psi_{\eta-(2 i+1)}}, \eta=0,1,2, \cdots$. where $a, b_{i}, \alpha$ and $\beta$ are positive real numbers for $i=0,1, \cdots, k$, and the initial conditions $\psi_{-j}, \psi_{-j+1}, \cdots, \psi_{0}$ are randomly positive real numbers where $j=2 k+1$. Accordingly, we consider the stability, boundedness and periodicity of the solutions of this recursive sequence. Indeed, we give some interesting counter examples in order to verify our strong results.
\end{abstract}

\section{Keywords}

Difference Equation, Stability, Boundedness, Globale Stability and Periodicity

\section{Introduction}

The global asymptotic behavior of the solutions and oscillation of solution are two such qualitative properties which are very important for applications in many areas such as control theory, mathematical biology, neural networks, etc. It is impossible to use computer based (numerical) techniques to study the oscillation or the asymptotic behavior of all solutions of a given equation due to the global nature of these properties. Therefore, these properties have received the attention of several mathematicians and engineers.

Currently, much attention has given to study the properties of the solutions of the recursive sequences from scientists in various disciplines. Specifically, the topics dealt with include the following:

- Finding equilibrium points for the recursive sequences;

- Investigating the local stability of the solutions of the recursive sequences; 
- Finding conditions which insure that the solutions of the recursive sequences are bounded;

- Investigating the global asymptotic stability of the solutions of the recursive sequences;

- Finding conditions which insure that the solutions of equation are periodic with positive prime period two or more;

- Finding conditions for oscillation of solutions.

Closely related global convergence results were well-gained from these articles [1]-[25]. Khuong in [14] studied the dynamics the recursive sequences

$$
\psi_{\eta+1}=a+\left(\frac{\psi_{\eta-k}}{\psi_{\eta-r}}\right)^{p} .
$$

For further related and special cases of this difference equations see [4] [5] [6], [21] [22] [24].

Elsayed [9] studied the periodicity, the boundedness of the positive solution of the recursive sequences

$$
\psi_{\eta+1}=a+\frac{b \psi_{\eta-l}+c \psi_{\eta-k}}{c \psi_{\eta-l}+d \psi_{\eta-k}} .
$$

Abdelrahman [1] considered analytical investigation of the solution of the recursive sequence

$$
\psi_{\eta+1}=a \psi_{\eta-k}+\delta\left(\frac{b \psi_{\eta-l}}{c \psi_{\eta-k}+d \psi_{\eta-l}}\right)^{\alpha} .
$$

By new method, Elsayed [10] investigated the periodic solution of the equation

$$
\psi_{\eta+1}=\alpha+\beta \frac{\psi_{\eta}}{\psi_{\eta-1}}+\gamma \frac{\psi_{\eta-1}}{\psi_{\eta}} .
$$

Also, Moaaz [18] completed the results of [10].

In this work, we deal with some qualitative behaviour of the solutions of the recursive sequence

$$
\psi_{\eta+1}=a+\sum_{i=0}^{k} b_{i} \frac{\psi_{\eta-(2 i+1)}}{\alpha \psi_{\eta-2 i}+\beta \psi_{\eta-(2 i+1)}}, \eta=0,1,2, \cdots
$$

where $a, b_{i}, \alpha$ and $\beta$ are positive real numbers for $i=0,1, \cdots, k$, and the the initial conditions $\psi_{-j}, \psi_{-j+1}, \cdots, \psi_{0}$ are arbitrary positive real numbers where $j=2 k+1$.

In the next, we will and to many of the basic concepts. Before anything, the concept of equilibrium point is essential in the study of the dynamics of any physical system. A point $\bar{\psi}$ in the domain of the function $\Phi$ is called an equilibrium point of the equation

$$
\psi_{\eta+1}=\Phi\left(\psi_{\eta}, \psi_{\eta-1}, \cdots, \psi_{\eta-k}\right), \eta=0,1,2, \cdots
$$

if $\bar{\psi}$ is a fixed point of $\Phi[\Phi(\bar{\psi}, \bar{\psi}, \cdots, \bar{\psi})=\bar{\psi}]$. For a stability of equilibrium 
point, equilibrium point $\bar{\psi}$ of equation (2) is said to be locally stable if for all $\varepsilon>0$ there exists $\delta>0$ such that, if $\psi_{-v} \in(0, \infty)$ for $v=0,1, \cdots, k$ with $\sum_{i=0}^{k}\left|\psi_{-i}-\bar{\psi}\right|<\delta$. As well, $\bar{\psi}$ is said to be locally asymptotically stable if it is locally stable and there exists $\gamma>0$ such that, if $\psi_{-v} \in(0, \infty)$ for $v=0,1, \cdots, k$ with $\sum_{i=0}^{k}\left|\psi_{-i}-\bar{\psi}\right|<\gamma$, then $\lim _{\eta \rightarrow \infty} \psi_{\eta}=\bar{\psi}$. Also, $\bar{\psi}$ is said to be a global attractor if used for every $\psi_{-v} \in(0, \infty)$ for $v=0,1, \cdots, k$, we have $\lim _{\eta \rightarrow \infty} \psi_{\eta}=\bar{\psi}$. On the other hand, $\bar{\psi}$ is said to be unstable if it is not locally stable.

Finally, Equation (1.2) is called permanent and bounded if there exists numbers $r$ and $R$ with $0<r<R<\infty$ such that for any initial conditions $\psi_{-v} \in(0, \infty)$ for $v=0,1, \cdots, k$ there exists a positive integer $N$ which depends on these initial conditions such as $r<\psi_{\eta}<R$ for all $\eta \geq N$.

The linearized equation of Equation (1.1) about the equilibrium point $\hat{\psi}$ is

$$
y_{\eta+1}=\sum_{i=0}^{k} p_{i} y_{\eta-i}
$$

where

$$
p_{i}=\frac{\partial F}{\partial \psi_{\eta-i}}(\hat{\psi}, \hat{\psi}, \cdots, \hat{\psi}) .
$$

Theorem 1.1. [15] Assume that $p_{i} \in \mathbb{R}$ for $i=0,1, \cdots, k$. The +ve equilibrium of (1.1) is locally asymptotically stable if

$$
\left|p_{0}\right|+\left|p_{1}\right|+\cdots+\left|p_{k}\right|<1
$$

\section{Local Stability of Equation (1.1)}

The +ve equilibrium point of Equation (1.1) is

$$
\hat{\psi}=a+\sum_{i=0}^{k} \frac{b_{i} \hat{\psi}}{\alpha \hat{\psi}+\beta \hat{\psi}},
$$

and so,

$$
\hat{\psi}=a+\frac{B}{\alpha+\beta},
$$

where

$$
B=\sum_{i=0}^{k} b_{i}
$$

Let $f \in C\left((0, \infty)^{2 k+2},(0, \infty)\right)$ defined by

$$
f\left(u_{0}, u_{1}, \cdots, u_{2 k+1}\right)=a+\sum_{i=0}^{k} \frac{b_{i} u_{2 i+1}}{\alpha u_{2 i}+\beta u_{2 i+1}} .
$$

Therefore it follows that

$$
\frac{\partial f}{\partial u_{2 r}}=-\frac{b_{r} \alpha u_{2 r+1}}{\left(\alpha u_{2 r}+\beta u_{2 r+1}\right)^{2}}
$$

and 


$$
\frac{\partial f}{\partial u_{2 r+1}}=\frac{b_{r} \alpha u_{2 r}}{\left(\alpha u_{2 r}+\beta u_{2 r+1}\right)^{2}},
$$

for $r=0,1, \cdots, k$.

Theorem 2.1. Let $\hat{\psi}$ be $+v e$ equilibrium of Equation (1.1). If

$$
(\alpha-\beta) B<a(\alpha+\beta)^{2},
$$

than $\hat{\psi}$ is locally stable.

Proof. From (2.2) to (2.3), we obtain

$$
\frac{\partial f}{\partial u_{2 r}}(\hat{\psi}, \cdots, \hat{\psi})=-\frac{b_{r} \alpha}{(\alpha+\beta)(a(\alpha+\beta)+B)}=p_{2 r},
$$

and

$$
\frac{\partial f}{\partial u_{2 r+1}}(\hat{\psi}, \cdots, \hat{\psi})=\frac{b_{r} \alpha}{(\alpha+\beta)(a(\alpha+\beta)+B)}=p_{2 r+1},
$$

for $r=0,1, \cdots, k$. Thus, the linearized equation of (1.1) is

$$
y_{\eta+1}=p_{0} y_{\eta}+p_{1} y_{\eta-1}+\cdots+p_{2 k+1} y_{\eta-(2 k+1)} .
$$

It follows by Theorem 1.1 that Equation (1.1) is locally stable if

$$
\left|\frac{b_{0} \alpha}{(\alpha+\beta)}\right|+\left|\frac{b_{0} \alpha}{(\alpha+\beta) \rho}\right|+\cdots+\left|\frac{b_{k} \alpha}{(\alpha+\beta) \rho}\right|+\left|\frac{b_{k} \alpha}{(\alpha+\beta) \rho}\right|<1,
$$

where $\rho=(a(\alpha+\beta)+B)$, and hence,

$$
\frac{2 \alpha}{(\alpha+\beta) \rho} B<1 .
$$

Thus, we find

$$
2 \alpha B<(\alpha+\beta) \rho,
$$

and so,

$$
(\alpha-\beta) B<a(\alpha+\beta)^{2} .
$$

Hence, the proof is complete.

In order to verify and support our theoretical outcomes and discussions, in this concern, we investigate several interesting numerical examples.

Example 2.1. By Theorem 2.1, the +ve equilibrium Equation (1.1) with $a=2, k=2, b_{i}=0.3, \quad \beta=0.1$ and $\alpha=1$, is locally stable (see Figure 1).

\section{Global Stability of Equation (1.1)}

In the following theorem, we check into the global stability of the recursive sequence (1.1).

Theorem 3.1. The +ve equilibrium $\hat{\psi}$ of Equation (1.1) is global attractor if

$$
B=a(\alpha-\beta) .
$$




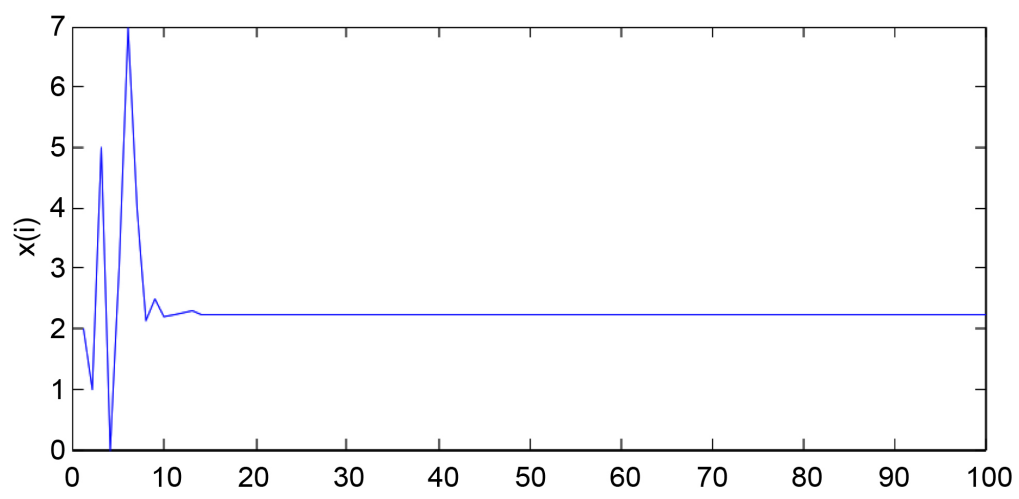

Figure 1. The stable solution corresponding to difference Equation (1.1).

Proof. We consider the function as follow:

$$
f\left(u_{0}, u_{1}, \cdots, u_{2 k+1}\right)=a+\sum_{i=0}^{k} \frac{b_{i} u_{2 i+1}}{\alpha u_{2 i}+\beta u_{2 i+1}} .
$$

From (2.2) and (2.3), we note that $f$ is increasing in $u_{2 r+1}$ and decreasing in $u_{2 r}$ for all $r=0,1, \cdots, k$. Suppose that $(\lambda, \mu)$ is a solution of the system

$$
\begin{aligned}
& \lambda=f(\mu, \lambda, \mu, \lambda, \cdots, \lambda) \\
& \mu=f(\lambda, \mu, \lambda, \mu, \cdots, \mu) .
\end{aligned}
$$

Then, we find

$$
\lambda=a+\sum_{i=0}^{k} b_{i} \frac{\lambda}{\alpha \mu+\beta \lambda},
$$

and

$$
\mu=a+\sum_{i=0}^{k} b_{i} \frac{\mu}{\alpha \lambda+\beta \mu} .
$$

Hence, we get

$$
\lambda=a+\frac{B \lambda}{\alpha \mu+\beta \lambda},
$$

and

$$
\mu=a+\frac{B \mu}{\alpha \lambda+\beta \mu} .
$$

By (3.1) and (3.2), we obtain

$$
\beta\left(\lambda^{2}-\mu^{2}\right)-(a \beta+B-a \alpha)(\lambda-\mu)=0 .
$$

Thus,

$$
(\lambda-\mu)(\beta(\lambda+\mu)-(a \beta+B-a \alpha))=0 .
$$

Since $B=a(\alpha-\beta)$, we have that $\mu=\lambda$. Hence, the proof of Theorem 3.1 is complete.

\section{Periodic Solutions}

In this section, we enumerate some basic facts concerning the existence of two 
period solutions.

Theorem 4.1. Equation (1.1) has prime period-two solutions if

$$
(a \beta+B-a \alpha)(\alpha-\beta)>4 a \alpha \beta .
$$

Proof. Assume that Equation (1.1) has a prime period-two solution

$$
\cdots, \rho, \sigma, \rho, \sigma, \rho, \sigma, \cdots
$$

We shall prove that condition (4.1) holds. From Equation (1.1), we see that

$$
\psi_{\eta}=\psi_{\eta-2}=\cdots=\psi_{\eta-2 k}=\sigma, \psi_{\eta+1}=\psi_{\eta-1}=\cdots=\psi_{\eta-(2 k+1)}=\rho,
$$

and hence,

$$
\rho=a+\frac{B \rho}{\alpha \sigma+\beta \rho}, \quad \sigma=a+\frac{B \sigma}{\alpha \rho+\beta \sigma} .
$$

Thus, we get

$$
\alpha \rho \sigma+\beta \rho^{2}=a \alpha \sigma+a \beta \rho+B \rho,
$$

and

$$
\alpha \rho \sigma+\beta \rho^{2}=a \alpha \sigma+a \beta \sigma+B \sigma .
$$

From (4.3) and (4.2), we have

$$
\beta\left(\rho^{2}-\sigma^{2}\right)=a \alpha(\sigma-\rho)+a \beta(\rho-\sigma)+B(\rho-\sigma) .
$$

Dividing $(\rho-\sigma)$, then we find

$$
(\rho+\sigma)=\frac{a \beta+B-a \alpha}{\beta}
$$

By combining (4.2) and (4.3), we obtain

$$
2 \alpha \rho \sigma+\beta\left(\rho^{2}+\sigma^{2}\right)=(a \alpha+a \beta+B)(\rho+\sigma) .
$$

Since $\rho^{2}+\sigma^{2}=(\rho+\sigma)^{2}-2 \rho \sigma$, we get

$$
\rho \sigma=\frac{a \alpha(a \beta+B-a \alpha)}{\beta(\alpha-\beta)} .
$$

Now, evident is that (4.4) and (4.5) that $\rho$ and $\sigma$ are both two positive distinct roots of the quadratic equation

$$
u^{2}+(\rho+\sigma) u+\rho \sigma=0 .
$$

Hence, we obtain

$$
\frac{(a \beta+B-a \alpha)}{\beta}>\frac{4 a \alpha}{\alpha-\beta},
$$

which has the same extent as

$$
(a \beta+B-a \alpha)(\alpha-\beta)>4 a \alpha \beta .
$$

Hence, the proof is complete.

The next numerical example is mimicry to enhance our results.

Example 4.1. By Theorem 4.1, Equation (1.1) with $\alpha=0.02, \beta=0.01$, $a=500, b_{0}=2, b_{1}=200$ and $b_{2}=20$, has prime period two solution (see 
Figure 2)

\section{Boundedness}

Theorem 5.1. Every solution of Equation (1.1) is bounded and persists.

Proof. Let $\left\{\psi_{\eta}\right\}_{\eta=-k}^{\infty}$ be a Solution (1.1), we can conclude from (1.1) that

$$
\psi_{\eta+1}=a+\sum_{i=0}^{k} b_{i} \frac{\psi_{\eta-(2 i+1)}}{\alpha \psi_{\eta-2 i}+\beta \psi_{\eta-(2 i+1)}}>a .
$$

Then

$$
\psi_{\eta}>a \text { for all } \eta>-(2 k+1) .
$$

Also, from Equation (1.1), we see that

$$
\begin{aligned}
\psi_{\eta+1} & =a+\sum_{i=0}^{k} b_{i} \frac{\psi_{\eta-(2 i+1)}}{\alpha \psi_{\eta-2 i}+\beta \psi_{\eta-(2 i+1)}} \\
& <a+\sum_{i=0}^{k} b_{i} \frac{\psi_{\eta-(2 i+1)}}{\beta \psi_{\eta-(2 i+1)}} \\
& =a+\sum_{i=0}^{k} \frac{b_{i}}{\beta}
\end{aligned}
$$

then,

$$
a<\psi_{\eta} \leq a+\frac{B}{\beta} \text { for all } \eta>-(2 k+1) .
$$

Thus, the solution is bounded and persists and the proof is complete.

Conclusion 1. In this paper, we study a asymptotic behavior of solutions of a general class of difference Equation (1.1). Our results extend and generalize to the earlier ones. Moreover, we obtain the next results.

- The +ve equilibrium point $\hat{\psi}$ of Equation (1.1) is local stable if $(\alpha-\beta) B<a(\alpha+\beta)^{2}$. Also, if $B=a(\alpha-\beta)$, then $\hat{\psi}$ is global attractor.

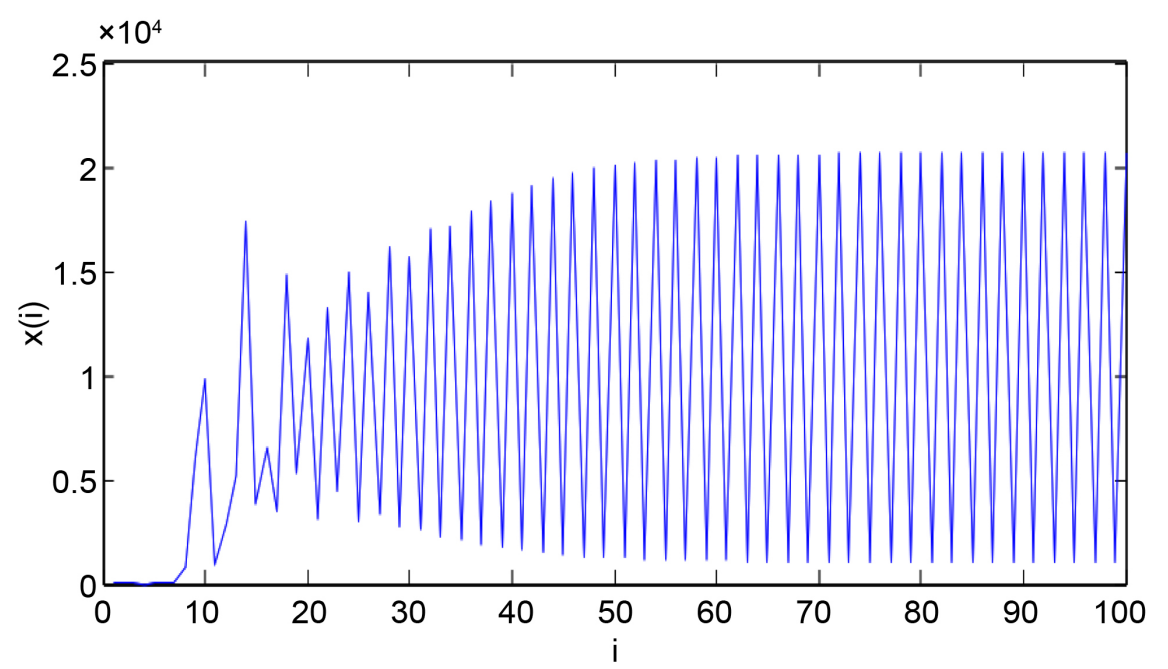

Figure 2. Prime period two solution of Equation (1.1). 
- Equation (1.1) has a prime period-two solutions if $(a \beta+B-a \alpha)(\alpha-\beta)>4 a \alpha \beta$.

- Every solution of (1.1) is bounded and persists.

\section{Acknowledgements}

The author is very grateful to the reviewers for their valuable suggestions and useful comments on this paper.

\section{References}

[1] Abdelrahman, M.A.E. and Moaaz, O. (2017) Investigation of the New Class of the Nonlinear Rational Difference Equations. Fundamental Research and Development International, 7, 59-72.

[2] Abdelrahman, M.A.E. and Moaaz, O. (2018) On the New Class of the Nonlinear Difference Equations. Electronic Journal of Mathematical Analysis and Applications, 6, 117-125.

[3] Agarwal, R. (1992) Difference Equations and Inequalities. Tteory, Methods and Applications, Marcel Dekker Inc., New York.

[4] Amleh, A.M., Grove, E.A., Georgiou, A. and Ladas, G. (1999) On the Recursive Sequence $\omega_{\eta+1}=\alpha+\frac{\omega_{\eta-1}}{\omega_{\eta}}$. The Journal of Mathematical Analysis and Applications, 233, 790-798. https://doi.org/10.1006/jmaa.1999.6346

[5] Berenhaut, K.S. and Stevic, S. (2006) The Behaviour of the Positive Solutions of the Difference Equation $\psi_{\eta}=a+\left(\psi_{\eta-2} / \psi_{\eta-1}\right)^{\delta}$. Journal of Difference Equations and Applications, 12, 909-918. https://doi.org/10.1080/10236190600836377

[6] Camouzis, E., DeVault, R. and Ladas, G. (2001) On the Recursive Sequence $\omega_{\eta+1}=-1+\omega_{\eta-1} / \omega_{\eta}$. Journal of Difference Equations and Applications, 7, 477-482. https://doi.org/10.1080/10236190108808283

[7] Elabbasy, E.M., EL-Metwally, H.A. and Elsayed, E.M. (2006) On the Difference Equation $\omega_{\eta+1}=a \omega_{\eta}-b \omega_{\eta} /\left(c \omega_{\eta}-d \omega_{\eta-1}\right)$. Advances in Difference Equations, 2006, 1-10. https://doi.org/10.1155/ADE/2006/82579

[8] Elaydi, S.N. (1996) An Introduction to Difference Equations, Undergraduate Texts in Mathematics. Springer, New York. https://doi.org/10.1007/978-1-4757-9168-6

[9] Elsayed, E.M. and El-Metwally, H.A. (2012) Qualitative Studies of Scalars and Systems of Difference Equations. Publisher LAP Lambert Academic, Saarbrücken.

[10] Elsayed, E.M. (2015) New Method to Obtain Periodic Solutions of Period Two and Three of a Rational Difference Equation. Nonlinear Dynamics, 79, 241-250. https://doi.org/10.1007/s11071-014-1660-2

[11] Elsayed, E.M. (2016) Dynamics and Behavior of a Higher Order Rational Difference Equation. The Journal of Nonlinear Sciences and Applications, 9, 1463-1474.

[12] Elsayed, E.M. (2010) On the Global Attractivity and the Periodic Character of a Recursive sequence. Opuscula Mathematica, 30, 431-446. https://doi.org/10.7494/OpMath.2010.30.4.431

[13] Grove, E.A. and Ladas, G. (2005) Periodicities in Nonlinear Difference Equations, Vol. 4. Chapman \& Hall/CRC, London.

[14] Khuong, V.V. (2008) On the Positive Nonoscillatory Solution of the Difference Eq- 
uations $\omega_{\eta+1}=\alpha+\left(\omega_{\eta-k} / \omega_{\eta-r}\right)^{p}$. Applied Mathematics-A Journal of Chinese Universities, 24, 45-48.

[15] Kulenovic, M.R.S. and Ladas, G. (2001) Dynamics of Second Order Rational Difference Equations with Open Problems and Conjectures. Chapman \& Hall/CRC, Florida. https://doi.org/10.1201/9781420035384

[16] El-Metwally, H., Ladas, G., Grove, E.A. and Voulov, H.D. (2001) On the Global Attractivity and the Periodic Character of Some Difference Equations. Journal of Difference Equations and Applications, 7, 837-850. https://doi.org/10.1080/10236190108808306

[17] Metwally, H.E., Grove, E.A. and Ladas, G. (2000) A Global Convergence Result with Applications to Periodic Solutions. Journal of Mathematical Analysis and Applications, 245, 161-170. https://doi.org/10.1006/jmaa.2000.6747

[18] Moaaz, O. (2016) Comment on New Method to Obtain Periodic Solutions of Period Two and Three of a Rational Difference Equation. Nonlinear Dynamics, 79, 241-250. https://doi.org/10.1007/s11071-016-3293-0

[19] Moaaz, O. and Abdelrahman, M.A.E. (2016) Behaviour of the New Class of the Rational Difference Equations. Journal of Mathematical Analysis and Applications, 4, 129-138.

[20] Moaaz, O. and Abdelrahman, M.A.E. (2017) On the Class of the Rational Difference Equations. International Journal of Advances in Mathematics, 2017, 46-55.

[21] Ocalan, O. (2014) Dynamics of the Difference Equation $\omega_{\eta+1}=p_{\eta}+\omega_{\eta-k} / \omega_{\eta}$ with a Period-Two Coefficient. Applied Mathematics and Computation, 228, 31-37. https://doi.org/10.1016/j.amc.2013.11.020

[22] Saleh, M. and Aloqeili, M. (2005) On the Difference Equation $\psi_{\eta+1}=A+\psi_{\eta} / \psi_{\eta-k}$. Applied Mathematics and Computation, 171, 862-869. https://doi.org/10.1016/j.amc.2005.01.094

[23] Stevic, S. (2005) On the Recursive Sequence $\psi_{\eta+1}=a+\beta \psi_{\eta-k} / f\left(\psi_{\eta}, \cdots, \psi_{\eta-k+1}\right)$. Taiwanese Journal of Mathematics, 9, 583-593.

[24] Sun, T. and Xi, H. (2007) On Convergence of the Solutions of the Difference Equation $\omega \eta+1=1+\psi_{\eta} / \psi_{\eta-k}$. Journal of Mathematical Analysis and Applications, 325, 1491-1494. https://doi.org/10.1016/j.jmaa.2006.02.080

[25] Zhang, L., Zhang, G. and Liu, H. (2005) Periodicity and Attractivity for a Rational Recursive Sequence. Journal of Applied Mathematics and Computing, 19, 191-201. https://doi.org/10.1007/BF02935798 